\title{
Healthy Adnexal Torsion and Pregnancy: A Case Report
}

Oumeyma Baroud*, Meriem Serraj Andaloussi, Amine Lamrissi, Karima Fichtali, Said Bouhya

Gynecology-Obstetrics Department HAROUCHI, University Hospital Center Ibn Rochd Casablanca

DOI: $10.36347 /$ sjmcr.2021.v09i01.012

| Received: 22.12.2020 | Accepted: 07.01.2021 | Published: 14.01.2021

*Corresponding author: Oumeyma Baroud

Abstract

Case Report

Healthy adnexal torsion during pregnancy is a rare occurrence. We present the case of a 38 -year-old patient who consulted for acute latero-pelvic pain during a 7-month pregnancy and who underwent an exploratory laparotomy which returned in favor of torsion on a healthy necrotic ovary. We performed a detorsion of the appendix with adnexectomy. The post-operative consequences were unremarkable. Torsion of the adnexa is an emergency that should not be ignored in the face of any acute pelvic pain in pregnant women. Conservative treatment is currently the gold standard and appropriate management is necessary to avoid possible maternal and fetal complications.

Key words: Torsion of the adnexa, healthy adnexa, rare pregnancy complication.

Copyright $\odot 2021$ The Author(s): This is an open-access article distributed under the terms of the Creative Commons Attribution 4.0 International License (CC BY-NC 4.0) which permits unrestricted use, distribution, and reproduction in any medium for non-commercial use provided the original author and source are credited.

\section{INTRODUCTION}

Torsion of the appendix is a rare pathology secondary to total or partial rotation of the appendix around its vascular axis. The occurrence of an appendix torsion in a healthy ovary is an even rarer situation [1]. It can occur during the three trimesters of pregnancy, although the increase in size of the uterus in the 2 nd and 3rd trimester reduces the mobility of the adnexa [2].

The interest of this situation lies in its diagnostic difficulty, and in the choice of the therapeutic attitude to adopt. We present the case of an appendix torsion in a healthy ovary occurring in the second trimester of pregnancy.

\section{Patient and observation}

Mrs. N.L, 38 years old, third gesture third par, without significant medical or surgical pathological history, consults the emergency room for paroxysmal left lateral pelvic pain evolving for 2 days, on a presumed pregnancy at 7 months. The admission examination found a conscious patient with a GCS at 15 , hemodynamically and respiratory stable: BP 12 / 7mmHg, HR: 90 beats / min, FR 15 cycles / min, afebrile at $37^{\circ}$. Abdominal examination finds right latero-uterine tenderness. At the gynecological examination; speculum: the cervix is macroscopically normal, no metrorrhagia or leucorrhoea and vaginal examination the cervix is long closed posteriorly. Abdomino-pelvic ultrasound shows an active monofetal pregnancy estimated at 28 weeks of amenorrhea; the two kidneys are well differentiated, with regular contours, without visible lesions. We also find in the right latero-uterine a slightly heterogeneous echogenic image with anechoic zones, $68 \times 50 \mathrm{~mm}$ without Doppler taking, suggesting a large twisted ovary. The patient underwent a median umbilical laparotomy, with exploration of an entirely necrotic left appendix, twisted by two turns (figure 1); the left dinghy was normal. The decision was therefore to perform a detorsion of the appendix, without recovery (Figure 2), hence the decision to complete with an adnexectomy and implementation of a utero-relaxing treatment postoperatively. The postoperative follow-up was simple, both obstetrically and surgically, with a good prognosis for vaginal delivery at term.

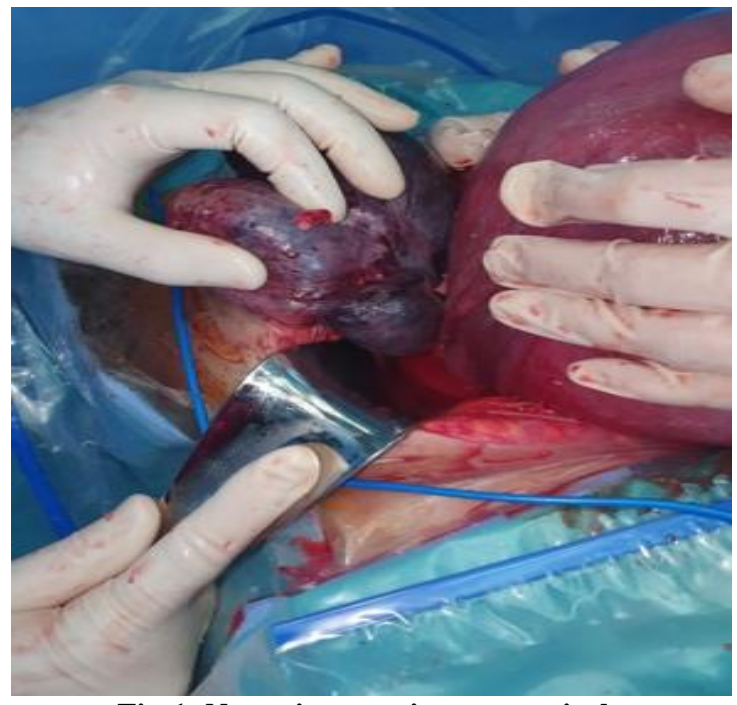

Fig-1: Necrotic ovary intraoperatively 


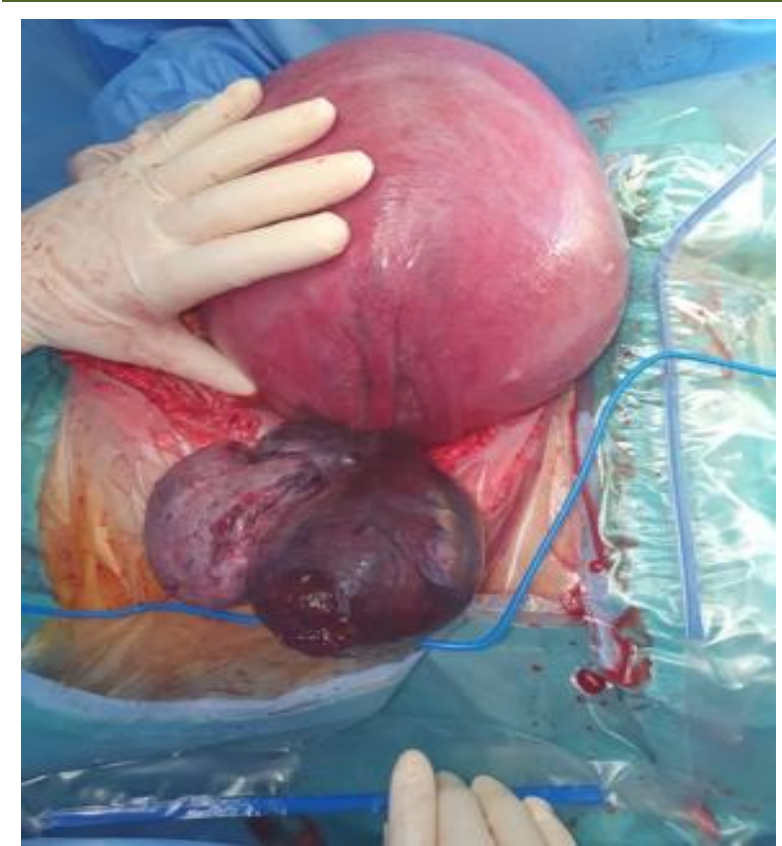

Fig-2: Intraoperative image after untwisting

\section{DiSCUSSION}

Adnexal torsion during pregnancy is a rare emergency. Its incidence is 3 to 5 per 10,000 pregnancies [3,4]. Between 8 and $28 \%$ of torsions occur during pregnancy, mostly in the first trimester but can be diagnosed at any age during pregnancy [5]. Usually the torsion occurs on a pathological ovary (malignant or benign tumor). Symptoms are generally characterized by sudden lateral pelvic pain $(98 \%$ of cases), associated with nausea and vomiting (78\% of cases) and a known adnexal mass (82\% of cases), From the second trimester, the absence of defense is possible, the appendages being located behind the gravid uterus. The torsion most often predominates on the right side and is favored by benign lesions of the ovary (dermoid cyst in $60 \%$ of cases) [2]. Its diagnosis during pregnancy is complicated because it requires the elimination of classic differential diagnoses but also those that may be linked to pregnancy. In addition, both clinical examination and imaging examinations become more difficult due to the uterine volume and the concomitant ascension of the ovary into the abdominal cavity. Torsion of the adnexa can cause uterine contractions, leading to a risk of miscarriage (early or late) or premature delivery depending on the age of pregnancy. The biological assessment is not specific, we can have hyperleukocytosis or an increase in the Creactive protein, indicating an inflammatory process. The ultrasound, even if it is poor in positive signs of torsion, remains the reference examination. It makes it possible to rule out differential diagnoses, and to search for factors that may promote torsion as well as indirect signs of ischemia. The interruption of the venous flow causes a reactive edema which is identifiable by the increase in ovarian volume compared to the contralateral appendix [6, 7]. Moreover, the increase in the number of cortical follicles is a non-specific aspect. but which has been found multiple times in the case of torsion in a healthy ovary [1]. The usefulness of ovarian vessel doppler remains controversial. Although the absence of a Doppler signal confirms the absence of blood flow and therefore the torsion, the reverse is false [8]. MRI is a satisfactory complementary exploration technique in pregnant women, who have a greater precision than ultrasound [9]. The combination of Doppler and MRI allows a better diagnostic approach but should not delay surgical management.

Torsion of the appendix is a real surgical emergency. Currently, laparoscopy is recommended for a gestational age of less than 17 weeks with, however, safety instructions to be observed: the preferential use of an open-laparoscopy, an insufflation pressure between 8 and $12 \mathrm{mmHg}$, an adapted position of the trocars and finally a gentle mobilization of the uterus. Conservative or radical treatment is decided according to the appearance of the appendix 10 minutes after detorsion [10]: for stages 1 and 2 (lesions with total or partial recovery after detorsion), conservative treatment is recommended, allowing functional recovery in $90 \%$ of cases. For stage 3 (necrotic, black and friable lesions without recovery after untwisting), adnexectomy appears preferable. For other authors, the great capacity for functional recovery of the ovarian tissue justifies being conservative even in the presence of an appendix of doubtful vitality $[10,11]$. Ovarian pexy is essential when there is a malformation of the ovarian ligaments, or an immediate recurrence of the torsion [12].

\section{CONCLUSION}

Classically described on pathological ovaries or tubes, adnexal torsion can however rarely occur in healthy ovaries [13].

Diagnosis remains difficult, especially during pregnancy and in the presence of a healthy appendix. Indeed, the clinical picture is not very specific, the paraclinical examinations are not very reliable for the positive diagnosis but retain a place in order to eliminate the differential diagnoses and to look for an adnexal pathology. It is important to assess the benefit risk ratio of performing a diagnostic laparoscopy while respecting the safety instructions for abdominal pain during pregnancy resistant to level III analgesics so as not to err on the diagnostic level by increasing the number of paraclinical examinations [2]. The operative procedure must be conservative and consists of untwisting the appendix, ovariopexy must not be systematic. The pregnancy prognosis is generally favorable, a few cases of growth retardation and premature delivery have been described.

\section{REFERENCES}

1. Guennoun A, Krimou Y, Mamouni N, Errarhay S, Bouchikhi C, Banani A. Torsion d'annexe saine et 
grossesse: à propos d'un cas. The Pan African Medical Journal. 2017;27.

2. Mattuizzi A, Camberlein C, Coatleven F, Horovitz J. Torsion d'annexe saine et grossesse: à propos d'un cas. Journal de Gynécologie Obstétrique et Biologie de la Reproduction. 2016 Jan 1;45(1):923.

3. Zanetta G, Mariani E, Lissoni A, Ceruti P, Trio D, Strobelt N, Mariani S. A prospective study of the role of ultrasound in the management of adnexal masses in pregnancy. BJOG: An International Journal of Obstetrics \& Gynaecology. 2003 Jun;110(6):578-83.

4. Kumari I, Kaur S, Mohan H, Huria A. Adnexal masses in pregnancy: A 5- year review. Australian and New Zealand journal of obstetrics and gynaecology. 2006 Feb;46(1):52-4.

5. Boughizane S, Naifer R, Hafsa A, Chaieb A, Hidar S, Lassouad L, Saidi H, Bibi M, Khairi H. Laparoscopic management of adnexal tumors after the first trimester of pregnancy. Journal de gynecologie, obstetrique et biologie de la reproduction. 2004 Jun;33(4):319-24.

6. Bellah RD, Griscom NT. Torsion of normal uterine adnexa before menarche: CT appearance. American Journal of Roentgenology. 1989 Jan 1;152(1):123-4.

7. Tepper R, Zalel Y, Goldberger S, Cohen I, Markov S, Beyth Y. Diagnostic value of transvaginal color
Doppler flow in ovarian torsion. European Journal of Obstetrics \& Gynecology and Reproductive Biology. 1996 Sep 1;68:115-8.

8. Abu-Musa A, Nassar A, Usta I, Khalil A, Hussein M. Laparoscopic unwinding and cystectomy of twisted dermoid cyst during second trimester of pregnancy. The Journal of the American Association of Gynecologic Laparoscopists. 2001 Aug 1;8(3):456-60.

9. Mage G, Canis M, Manhes H, Pouly JL, Bruhat MA. Laparoscopic management of adnexal torsion. A review of 35 cases. The Journal of reproductive medicine. 1989 Aug;34(8):520-4.

10. Bider D, Mashiach S, Dulitzky M, Kokia E, Lipitz S, Ben-Rafael ZI. Clinical, surgical and pathologic findings of adnexal torsion in pregnant and nonpregnant women. Surgery, gynecology \& obstetrics. 1991 Nov;173(5):363-6.

11. Way S. Ovarian cystectomy of twisted cysts. Lancet (London, England). 1946 Jul 13;2(6411):47.

12. N. Dh et J. Pj, « Torsion of the adnexa. », Clin. Obstet. Gynecol. 1985; 28(2) : 375- 380.

13. Vincens C, Rathat G, Boulot P, Giacalone PL. Recurrent adnexal torsion with normal ovarian, in the second and third trimesters of pregnancy. Gynecologie, obstetrique \& fertilite. 2010;38(78):486. 